\title{
Electromagnetic Wave Behaviour in Uniaxial Magnetodielectric Media of $1+1>2$ Type
}

\author{
J. Kapelewski AND A. DUKata* \\ Faculty of Electronics, Military University of Technology \\ S. Kaliskiego 2, 00-908 Warsaw, Poland
}

\begin{abstract}
In the paper a phenomenological approach is proposed to polarized electromagnetic wave propagation in magnetodielectric media, composed of piezoelectric and magnetostrictive anisotropic monolayers. The composite materials consisting of a system of such bimorph films have recently attracted much interest, because they can exhibit a much stronger magnetoelectric effect in a wide temperature range than the single phase multiferroic materials. In the paper dispersion equations given in a common form for both TE and TM modes are derived for long wave approximation. The equations can be of use in evaluation of directional dependence for particular polarizations, which is of particular interest in designing substrate for miniaturized polarimetric antenna, especially useful for modern synthetic aperture radar systems.
\end{abstract}

PACS numbers: 41.20.Jb, 43.20.Mv, 03.50.De

\section{Introduction}

In recent years, there has been increasing interest in the development of new materials with characteristics which may not be found in nature. Examples are metamaterials, which are structured composite materials with unique electromagnetic properties mainly due to the interaction of electromagnetic (EM) waves with periodically modified structure of conventional materials. They have a broad range of applications including artificial dielectrics, antenna structures, microwave components and composite materials. Applications of metamaterials in antenna design have been considered in recent years by several authors $[1-4]$. The electromagnetic analysis and optimization based on the effective medium approximations of artificial materials is very convenient since it deals with only a few bulk medium parameters instead of a large number of parameters fully describing a discrete structure. Effective medium parameters of composite electromagnetic materials used in such devices can be obtained through various homogenization methods. At microwave frequencies where the wavelength is much greater than at optical frequencies it is possible to construct a kind of composite superlattices having either isotropic or anisotropic properties. This paper presents EM wave propagation in an infinite periodic structure formed by alternating magnetic and dielectric layers of thickness $a_{1}$ and $a_{2}$ as illustrated in Fig. 1. Each of layers is characterized by uniaxial dyadics $\bar{\varepsilon}$ and $\bar{\mu}$.

One can discriminate two main kinds of "composite ef-

* corresponding author; e-mail: andrzej.dukata@wel.wat.edu.pl

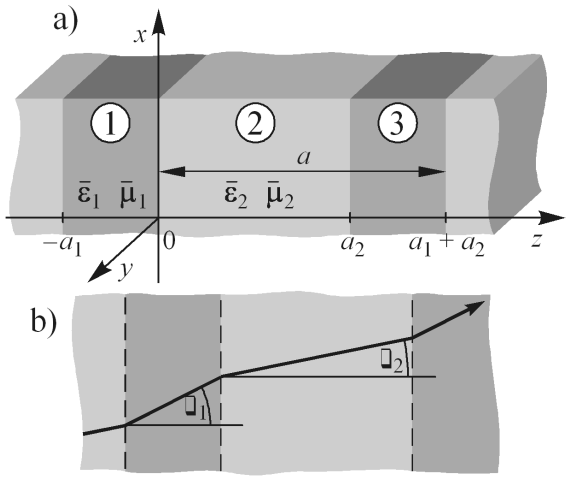

Fig. 1. An artificial anisotropic magnetodielectric medium.

fects" for planar EM waves. First, sometimes called the " $0+0>0$ composite effect" (see e.g. [5]) means that "new" materials property can be produced in the composites via the interaction between constituent phases. It is important and very prospective way to design and explore new materials properties with materials existing in nature. In the present paper we focus on the study of the alternative " $1+1>2$ composite effect" due to which, some performance of the composites can also be enhanced or improved as compared with the monophase materials.

The properties of the constituent phases are often assumed to be known. The question is then how to deduce the effective dynamical properties of the medium from the properties of the constituent phases. 
Before considering propagation in such a structure, some points of the theory of plane wave propagation in a homogeneous uniaxial medium will be briefly reviewed for later comparison.

\section{Propagation in a homogeneous uniaxial medium}

Uniaxial medium is a special case of the most general linear medium, whose constitutive equations are of the form

$$
\boldsymbol{D}=\varepsilon_{0} \overline{\boldsymbol{\varepsilon}} \cdot \boldsymbol{E}, \quad \boldsymbol{B}=\mu_{0} \overline{\boldsymbol{\mu}} \boldsymbol{H} .
$$

As a microwave electromagnetic material it can be well represented by a class of multiferroic (magnetodielectric) media. Due to axial symmetry of the medium $\bar{\varepsilon}$ and $\overline{\boldsymbol{\mu}}$ are uniaxial dyadics

$$
\overline{\boldsymbol{\varepsilon}}=\varepsilon_{t} \hat{\boldsymbol{x}} \hat{\boldsymbol{x}}+\varepsilon_{t} \hat{\boldsymbol{y}} \hat{\boldsymbol{y}}+\varepsilon_{z} \hat{\boldsymbol{z}} \hat{\boldsymbol{z}}, \quad \overline{\boldsymbol{\mu}}=\mu_{t} \hat{\boldsymbol{x}} \hat{\boldsymbol{x}}+\mu_{t} \hat{\boldsymbol{y}} \hat{\boldsymbol{y}}+\mu_{z} \hat{z} \hat{\boldsymbol{z}} .
$$

The fields are assumed to have a harmonic time dependence $\exp (\mathrm{j} \omega t)$. Since the medium is isotropic in the $x y$ plane, to study plane-wave propagation, without loss of generality we consider $2 \mathrm{D}$ problem with $\partial / \partial y=0$. With the mentioned constitutive relations and Maxwell equations

$$
\nabla \times \boldsymbol{E}=\mathrm{j} \omega \boldsymbol{B}, \quad \nabla \times \boldsymbol{H}=-\mathrm{j} \omega \boldsymbol{D},
$$

it is readily concluded that electromagnetic fields can be separated into TE $\left(E_{y}, H_{x}, H_{z}\right)$ and TM $\left(H_{y}, E_{x}, E_{z}\right)$ polarizations. For TE-polarization, we have

$$
\begin{aligned}
& \frac{\partial^{2} E_{y}}{\partial z^{2}}+\frac{\mu_{t}}{\mu_{z}} \frac{\partial^{2} E_{y}}{\partial x^{2}}+\frac{\omega^{2}}{c^{2}} \mu_{t} \varepsilon_{t} E_{y}=0, \\
& H_{x}=\mathrm{j} \frac{1}{\omega \mu_{0} \mu_{t}} \frac{\partial E_{y}}{\partial z}, \quad H_{z}=-\mathrm{j} \frac{1}{\omega \mu_{0} \mu_{z}} \frac{\partial E_{y}}{\partial x}
\end{aligned}
$$

and for TM-polarization

$$
\begin{aligned}
& \frac{\partial^{2} H_{y}}{\partial z^{2}}+\frac{\varepsilon_{t}}{\varepsilon_{z}} \frac{\partial^{2} H_{y}}{\partial x^{2}}+\frac{\omega^{2}}{c^{2}} \mu_{t} \varepsilon_{t} H_{y}=0, \\
& E_{x}=-\mathrm{j} \frac{1}{\omega \varepsilon_{0} \varepsilon_{t}} \frac{\partial H_{y}}{\partial z}, \quad E_{z}=\mathrm{j} \frac{1}{\omega \varepsilon_{0} \varepsilon_{z}} \frac{\partial H_{y}}{\partial x} .
\end{aligned}
$$

In the short notation, the propagation of EM wave can be expressed by the scalar wave equation

$$
\begin{gathered}
\left(\alpha \frac{\partial^{2}}{\partial x^{2}}+\frac{\partial^{2}}{\partial z^{2}}+k_{t}^{2}\right) \psi(x, z)=0, \\
\psi=\left\{\begin{array}{l}
E_{y} \text { for TE mode, } \\
H_{y} \text { for TM mode }
\end{array}\right.
\end{gathered}
$$

where

$$
\alpha=\left\{\begin{array}{cl}
\alpha_{-}=\mu_{t} / \mu_{z} & \text { for TE mode, } \\
\alpha_{+}=\varepsilon_{t} / \varepsilon_{z} & \text { for TM mode }
\end{array}\right.
$$

and $k_{t}^{2}=\left(\omega^{2} / c^{2}\right) \mu_{t} \varepsilon_{t}$. When plane wave solution $\psi=$ $\psi_{0} \exp (\mathrm{j} q \hat{\boldsymbol{n}} \cdot \boldsymbol{r})$ with direction of propagation defined by $\hat{\boldsymbol{n}}=\hat{\boldsymbol{x}} \sin \theta+\hat{\boldsymbol{z}} \cos \theta$ is substituted to (5), one obtains

$$
q_{ \pm}^{2}=\frac{k_{t}^{2}}{\alpha_{ \pm} \sin ^{2} \theta+\cos ^{2} \theta} .
$$

For a nonmagnetic medium $\left(\mu_{z}=\mu_{t}=1\right)$, the solution $q_{-}=k_{t}$ corresponds to the ordinary wave and the solution $q_{+}$corresponds to an extraordinary wave. The wave corresponding to $q_{-}$may be called transverse electric (TE), because the wave sees only the transverse component of the $\bar{\varepsilon}$ dyadic. For similar reasons, the other solution corresponding to $q_{+}$can be called transverse magnetic (TM).

$>$ From Eq. (6) it is readily seen that the axis of the medium corresponding to $\theta=0$ is the optical axis, because in this case the two solutions coincide

$$
q_{ \pm}^{2}=k_{t}^{2}=\left(\omega^{2} / c^{2}\right) \mu_{t} \varepsilon_{t} .
$$

Obviously, the fields $\boldsymbol{E}$ and $\boldsymbol{H}$ are polarized transverse to the axis $\hat{\boldsymbol{z}}$, thus the waves see the medium as isotropic with the transverse parameters $\varepsilon_{t}, \mu_{t}$.

In the transverse propagation $\theta=\pi / 2$, we can write

$$
q_{+}^{2}=k_{t}^{2} \frac{\varepsilon_{z}}{\varepsilon_{t}}=\frac{\omega^{2}}{c^{2}} \mu_{t} \varepsilon_{z}, \quad q_{-}^{2}=k_{t}^{2} \frac{\mu_{z}}{\mu_{t}}=\frac{\omega^{2}}{c^{2}} \varepsilon_{t} \mu_{z} .
$$

\section{Propagation in a stacked uniaxial magnetodielectric sheet medium}

Structures consisting of a periodic array of magnetodielectric composite materials provide important advantages, when used as substrates for planar antennas. These substrates are used to miniaturize antennas while maintaining a relatively high band width and efficiency.

The magnetodielectric slabs of the structure analyzed in this paper are uniaxial with parallel axes, infinite and isotropic in the $x z$ plane medium. Each unit cell contains two regions as illustrated in Fig. 1a.

Each slab of thickness $a_{1}$ and material parameters given by dyadics $\bar{\varepsilon}_{1}$ and $\bar{\mu}_{1}$ is separated by a slab of thickness $a_{2}$ and dyadics $\bar{\varepsilon}_{2}$ and $\overline{\boldsymbol{\mu}}_{2}$.

The EM waves for the electric and magnetic polarizations considered locally in one unit cell (consisting of area 1 and 2) must satisfy the scalar wave equation

$$
\left(\alpha_{n} \frac{\partial^{2}}{\partial x^{2}}+\frac{\partial^{2}}{\partial z^{2}}+k_{n t}^{2}\right) \psi_{n}(x, z)=0,
$$

where the subscript $n$ denotes 1 for $-a_{1} \leq z \leq 0$ and 2 for $0 \leq z \leq a_{2}\left(a_{1}+a_{2}=a\right)$.

Solution to (7) is found to be

$$
\psi_{n}(x, z)=\psi_{n}(z) \exp (\mathrm{j} s x),
$$

where

$$
\begin{aligned}
& \psi_{n}(z)=A_{n} \exp \left(\mathrm{j} k_{n} z\right)+B_{n} \exp \left(-\mathrm{j} k_{n} z\right) \text { and } \\
& k_{n}^{2}+\alpha_{n} s^{2}=k_{n t}^{2} .
\end{aligned}
$$

It is worth to note that after introducing to the description the incidence angle $\theta_{1}$ and refraction angle $\theta_{2}$ (Fig. 1b) a generalized form of Snell's law takes the form $s=q_{1} \sin \theta_{1}=q_{2} \sin \theta_{2}$, where $q_{n}^{2}=k_{n t}^{2} /\left(\alpha_{n} \sin ^{2} \theta_{n}+\right.$ $\cos ^{2} \theta_{n}$ ) (see Eq. (6)).

Using the Floquet-Bloch theorem the solutions $\psi_{n}(z)$ are of the general form $\psi_{n}(z)=u(z) \exp \left(\mathrm{j} k_{z} z\right)$ where $u(z)$ is a periodic function with a period $a: u(z-a)=$ $u(z)$. The Bloch wave number $k_{z}$ can be treated as a free 
parameter in the range $-\pi / a \leq k_{z} \leq \pi / a$. If $\psi_{3}(z)=$ $u(z) \exp \left(\mathrm{j} k_{z} z\right)$ then

$$
\begin{aligned}
& \psi_{1}(z-a)=u(z-a) \exp \left(\mathrm{j} k_{z}(z-a)\right) \\
& \quad=u(z) \exp \left(\mathrm{j} k_{z} z\right) \exp \left(-\mathrm{j} k_{z} a\right)=\psi_{3}(z) \exp \left(-\mathrm{j} k_{z} a\right)
\end{aligned}
$$

and using (8)

$$
\begin{aligned}
& \psi_{3}(z)=\exp \left(\mathrm{j} k_{z} l\right) \psi_{1}(z-a) \\
& \quad=\exp \left(\mathrm{j} k_{z} a\right)\left[A_{1} \exp \left(\mathrm{j} k_{1}(z-a)\right)\right. \\
& \left.\quad+B_{1} \exp \left(-\mathrm{j} k_{1}(z-a)\right)\right] .
\end{aligned}
$$

The TE-polarization boundary conditions are continuity of $E_{y}$ and $H_{x}$, then at $z=0$ and $z=a_{2}$ we have (see Eq. (2)):

$$
\begin{aligned}
& \psi_{1}(0)=\psi_{2}(0), \quad \frac{\psi_{1}^{\prime}(0)}{\mu_{1 t}}=\frac{\psi_{2}^{\prime}(0)}{\mu_{2 t}}, \quad \psi_{2}\left(a_{2}\right)=\psi_{3}\left(a_{2}\right), \\
& \frac{\psi_{2}^{\prime}\left(a_{2}\right)}{\mu_{1 t}}=\frac{\psi_{3}^{\prime}\left(a_{2}\right)}{\mu_{2 t}} .
\end{aligned}
$$

Next we substitute $\psi_{3}$ from Eq. (9) and $\psi_{1}, \psi_{2}$ from Eq. (8) into Eq. (10). It yields four relations

$$
\begin{aligned}
& A_{1}+B_{1}=A_{2}+B_{2}, \\
& k_{1}\left(A_{1}-B_{1}\right) \mu_{1 t}^{-1}=k_{2}\left(A_{2}-B_{2}\right) \mu_{2 t}^{-1}, \\
& A_{2} \exp \left(\mathrm{j} k_{2} a_{2}\right)+B_{2} \exp \left(-\mathrm{j} k_{2} a_{2}\right) \\
& \quad=\exp \left(\mathrm{j} k_{z} a\right)\left[A_{1} \exp \left(\mathrm{j} k_{1}\left(a_{2}-a\right)\right)\right. \\
& \left.\quad+B_{1} \exp \left(-\mathrm{j} k_{1}\left(a_{2}-a\right)\right)\right], \\
& k_{2}\left[A_{2} \exp \left(\mathrm{j} k_{2} a_{2}\right)-B_{2} \exp \left(-\mathrm{j} k_{2} a_{2}\right)\right] \mu_{2 t}^{-1} \\
& \quad=\exp \left(\mathrm{j} k_{z} a\right) k_{1}\left[A_{1} \exp \left(\mathrm{j} k_{1}\left(a_{2}-a\right)\right)\right. \\
& \left.\quad-B_{1} \exp \left(-\mathrm{j} k_{1}\left(a_{2}-a\right)\right)\right] \mu_{1 t}^{-1} .
\end{aligned}
$$

This set of four linear homogeneous equations in $A_{1}, B_{1}$, $A_{2}$, and $B_{2}$ coefficients can be solved only if its determinant is zero

$$
\Delta=\left|\begin{array}{cccc}
1 & 1 & -1 & -1 \\
k_{1} \mu_{2 t} & -k_{1} \mu_{2 t} & -k_{2} \mu_{1 t} & k_{2} \mu_{1 t} \\
\mathrm{e}^{\mathrm{j}\left(k_{z} a-k_{1} a_{1}\right)} & \mathrm{e}^{\mathrm{j}\left(k_{z} a+k_{1} a_{1}\right)} & -\mathrm{e}^{\mathrm{j} k_{2} a_{2}} & -\mathrm{e}^{-\mathrm{j} k_{2} a_{2}} \\
k_{1} \mu_{2 t} \mathrm{e}^{\mathrm{j}\left(k_{z} a-k_{1} a_{1}\right)} & -k_{1} \mu_{2 t} \mathrm{e}^{\mathrm{j}\left(k_{z} a+k_{1} a_{1}\right)} & -k_{2 z} \mu_{1 t} \mathrm{e}^{\mathrm{j} k_{2} a_{2}} & k_{2 z} \mu_{1 t} \mathrm{e}^{-\mathrm{j} k_{2} a_{2}}
\end{array}\right|=0 .
$$

Equation (11) is equivalent to

$$
\begin{aligned}
& \cos \left(k_{1} a_{1}\right) \cos \left(k_{2} a_{2}\right)-\frac{1}{2}\left(\frac{\mu_{1 t} k_{2}}{\mu_{2 t} k_{1}}+\frac{\mu_{2 t} k_{1}}{\mu_{1 t} k_{2}}\right) \\
& \times \sin \left(k_{1} a_{1}\right) \sin \left(k_{2} a_{2}\right)=\cos \left(k_{z} a\right) .
\end{aligned}
$$

The application of duality to (11) determines the following transcendental equation for the TM-polarization case:

$$
\begin{aligned}
& \cos \left(k_{1} a_{1}\right) \cos \left(k_{2} a_{2}\right)-\frac{1}{2}\left(\frac{\varepsilon_{1 t} k_{2}}{\varepsilon_{2 t} k_{1}}+\frac{\varepsilon_{2 t} k_{1}}{\varepsilon_{1 t} k_{2}}\right) \\
& \times \sin \left(k_{1} a_{1}\right) \sin \left(k_{2} a_{2}\right)=\cos \left(k_{z} a\right) .
\end{aligned}
$$

Formulae similar to Eq. (13) were derived in [6], however, only for the case of nonmagnetic and isotropic layers.

\section{Asymptotic solutions}

To obtain the dispersion relations we expand Eq. (12) and Eq. (13) in the Taylor series as a function of small parameters $k_{1} a_{1}, k_{2} a_{2}$ and $k_{z} a$. Using Eq. (6) and Eq. (8) after some manipulations in Mathematica we have for both TE and TM modes

$$
\begin{aligned}
& k_{z}^{2}=\frac{\omega^{2}}{c^{2}}\left(f_{1} \alpha_{1} \beta+f_{2} \alpha_{2}\right) \\
& \times \frac{\mu_{1 t} \varepsilon_{1 t} f_{1} \alpha_{2}+\mu_{2 t} \varepsilon_{2 t} f_{2} \alpha_{1} \beta\left[1+\left(\alpha_{1}-\alpha_{2}\right) \tan ^{2} \theta_{1}\right]}{\beta \alpha_{1} \alpha_{2}\left(1+\alpha_{1} \tan ^{2} \theta_{1}\right)},
\end{aligned}
$$

where $f_{1}=a_{1} / a$ and $f_{2}=a_{2} / a$ denote the volume frac- tions of the materials, $\theta_{1}$ is an angle of propagation in medium 1 and

$$
\beta= \begin{cases}\mu_{1 z} / \mu_{2 z} & \text { for TE mode, } \\ \varepsilon_{1 z} / \varepsilon_{2 z} & \text { for TM mode. }\end{cases}
$$

Let us note that the resulting dispersion equation Eq. (14) has the same form for both modes in consideration. As before, the $z$-axis is the optical axis because the Bloch wave number $k_{z}$ :

$$
k_{z}^{2}=\left(\omega^{2} / c^{2}\right)\left(f_{1} \varepsilon_{1 t}+f_{2} \varepsilon_{2 t}\right)\left(f_{1} \mu_{1 t}+f_{2} \mu_{2 t}\right)
$$

is the same for both TE and TM modes. It can be readily seen by putting $\theta_{1}$ in Eq. (14).

\section{Conclusions}

The dispersion equation for plane wave propagation is evaluated for a uniaxial medium composed of stacked magnetodielectric anisotropic layers. In this kind of medium there are two wave numbers $\left(k_{z}\right)$, which depend on the parameters of the particular layers and also on the propagation direction.

The two solutions coincide when the wave is propagating in the $z$ direction, which is the optical axis. The waves then see the medium as isotropic with four transverse medium parameters. In the slanting propagation the two solutions depend on all eight medium parameters. 


\section{Acknowledgments}

This paper is financed from sources for science in the years 2007-2010 in the frame of ordered scientific project PBZ-MNiSW-DBO-04/I/2007.

\section{References}

[1] H. Mosallaei, K. Sarabandi, IEEE Trans. Antennas Propagat. 55, 45 (2007).
[2] A. Dukata, J. Kapelewski, Acta Phys. Pol. 114, A-79 (2008).

[3] T. Pustelny, A. Opilski, B. Pustelny, Acta Phys. Pol. 114, A-181 (2008).

[4] A. Semichaevsky, A. Akyurtlu, Prog. Electromagn. Res. PIER 71, 129 (2007).

[5] C.W. Nan, Yuanhua Lin, Jin H. Huang, Ferroelectrics 280, 153 (2002).

[6] D. Sjöberg, J. Electromagn. Waves Appl. 20, 781 (2006). 